\title{
Exploratory Data Analysis on Stochastic Emissions Near-Field Scanning Measurements
}

\author{
José A. Oliva, Marco A. Azpúrua, Marc Pous and, \\ Ferran Silva \\ Grup de Compatibilitat Electromagnètica \\ Universitat Politècnica de Catalunya \\ Barcelona, Spain \\ email: jose.oliva@upc.edu
}

\author{
M H Baharuddin, C Smartt and, \\ David W. P. Thomas \\ George Green Institute for Electromagnetics Research \\ University of Nottingham \\ University Park, Nottingham NG7 2RD \\ email: dave.thomas@nottingham.ac.uk
}

\begin{abstract}
Recently, near-field scanning techniques for measuring and analysing stochastic emissions have been theoretically and experimentally studied and they are promising in terms of the characterization and classification radiated emissions in densely integrated technologies. Nonetheless, one of their drawbacks is the volume of data generated by time-domain acquisitions made across the scanning grid. Moreover, there are a multiplicity of sources of systematic errors that can pass unnoticed in measurements and that could compromise the accuracy of the data used for post-processing. This paper presents a preevaluation procedure that follows an exploratory data analysis approach for detecting such errors using an objective statistical criterion when applied to stochastic emissions of near-field scanning measurement data. Results are presented using real measurement data from the scanning of an Arduino Intel Galileo board, as has been reported in previous studies. By performing the proposed analysis, significant errors were detected in the measurement data. Final remarks on the importance of performing quality clearance in the measurement data from stochastic emissions assessments are provided.
\end{abstract}

Keywords-Near-field scanning, radiated emissions, stochastic fields, time-domain measurements

\section{INTRODUCTION}

Near-field measurements of equipment can be used to directly estimate the far-field radiation pattern [1] or equivalent sources can be derived to predict the radiation pattern [2]. The increased functionality and circuit density of electronic equipment, however, means that it is not possible to resolve individual current sources using near-field scans and the current sources are often incoherent without a common continuous phase reference and broadband. To measure such wide bandwidth complex fields it is necessary to treat them as stochastic fields and measure the time domain correlation of the near field using a two probe scanning technique [3]-[5]. From the correlation of the fields, it is possible to identify the direction of propagation and hence predict the field distribution at other distances or the far field [6].

However, such an approach is very challenging [5], [6]. If the spatial resolution as defined in [1] has to be used then this results in a very time consuming measurement process and the amount of data recorded is extremely large requiring a lot of computing resources for recording and analysing it. Several methods of reducing the complexity have been proposed such as using Principle Component analysis [7], [8] or a reduced set of reference points [9]. PCA is a purely data driven process with no prior knowledge of the source configuration. It therefore only offers a suitable method for data reduction but cannot provide a useful tool for reducing the measurements. The use of a reduced set of measurement points is potentially very useful but it is unclear how these points can be preselected. It is also possible to use the natural cyclostationary properties of the emissions real world devices to reduce the measurement time and computational burden [10].

During the exhaustive and time-consuming measurements performed by the dual-probe stochastic emissions near-field scanners there a multiplicity of factors of influence and sources of error that can undermine the accuracy of the measurements. It is, therefore, of substantial importance to examine the data before proceeding to further processing it, in operations such as calculating the correlation matrixes and propagating the fields [5], [6]. Incidentally, such preliminary evaluation of measurements has to be carried out automatically, objectively and without adding substantial delay to the calculation of the final processed results. In consequence, this paper proposes using the exploratory data analysis (EDA) techniques for looking at patters in the data that are characteristic of systematic errors in the measurements. Such an approach is defined using objective statistical tests and criterions that would allow an agile identification of problems in the data.

The paper is structured as follows. First, section II summarizes the theory and practice of the near-field scanning of stochastic field emissions, then section III describes in detail the approach followed in the EDA while section IV is devoted to present the results from the EDA applied on a particular set of measurements the results of which comprised by notable systematic errors. Next, a brief discussion is given in section $\mathrm{V}$ regarding the most prominent sources of error that must be taken into account, controlled and, avoided for performing accurate near-field scanning of stochastic field emissions measurements. Finally, the paper closes with the conclusions and a couple of remarks concerning aspects that must be further investigated for reliable and traceable measurements of the stochastic emissions using near-field scanning methods. 


\section{NEAR-FIELD SCANNING OF StOchastic FIELD EMISSIONS}

\section{A. Stochactic Electromagnetic Fields}

It has been shown in [4] that the power spectra of noisy signals can be defined from suitably time-windowed field correlations. Let a vector magnetic field at a point $x$ in the time domain $t$ be given by the function $\boldsymbol{h}(x, t)$ then a correlation function of the field distribution can be described by [4]:

$$
c_{h}\left(x_{1}, x_{2}, \tau\right)=\lim _{T \rightarrow \infty} \frac{1}{2 T} \int_{-\infty}^{\infty} \boldsymbol{h}_{T}\left(x_{1}, t\right) \boldsymbol{h}_{T}\left(x_{2}, t-\tau\right) d t,
$$

where $\boldsymbol{h}_{T}$ denotes the time-windowed signal, defined by

$$
\boldsymbol{h}_{T}(t)=\left\{\begin{array}{cc}
\boldsymbol{h}(t) & \text { for }-T<t<T \\
0 & \text { for } \quad|t| \geq T
\end{array}\right.
$$

For $x_{1} \neq x_{2}$ the function $c_{h}\left(x_{1}, x_{2} \tau\right)$ is the cross-correlation of the field and for $x_{1}=x_{2}$ it is the autocorrelation. The Fourier transform of the cross-correlation of the field is the correlation spectrum given by:

$$
\Gamma_{H}\left(x_{1}, x_{2}, \omega\right)=\int_{-\infty}^{\infty} c_{\mathrm{h}}\left(x_{1}, x_{2}, \tau\right) \exp (-j \omega \tau) d \tau,
$$

The correlation spectra can also be directly derived from the Fourier transform of the time-windowed fields $\boldsymbol{H}_{T}(x, \omega)$ using:

$$
\underline{\underline{\Gamma_{H}}}\left(x_{1}, x_{2}, \omega\right)=\lim _{T \rightarrow \infty} \frac{1}{2 T}\left\langle\boldsymbol{H}_{\mathrm{T}}\left(x_{1}, \omega\right) \boldsymbol{H}_{\mathrm{T}}^{*}\left(x_{2}, \omega\right)\right\rangle,
$$

where $\langle>$ denotes the forming of the ensemble average. The spectral magnetic energy density $W_{H}(x, \omega)$ is then given by

$$
W_{H}(x, \omega)=\frac{\mu}{2} \Gamma_{H}(x, x, \omega),
$$

where $\mu$ is the magnetic permeability of the medium. For the signal is cyclostationary then the mean and autocorrelation have to be periodic [11]. This the measured magnetic field must satisfy (6) and (7) for a cyclostationary period $T_{0}$ :

$$
\begin{gathered}
\langle\boldsymbol{h}(t)\rangle=\left\langle\boldsymbol{h}\left(t+T_{0}\right)\right\rangle, \\
\left\langle\boldsymbol{h}(t) \boldsymbol{h}^{*}(t-\tau)\right\rangle=\left\langle\boldsymbol{h}\left(t+T_{0}\right) \boldsymbol{h}^{*}\left(t+T_{0}-\tau\right)\right\rangle,
\end{gathered}
$$

The correlation dyadic, periodic in time $T_{0}$ can then be expanded into the Fourier series:

$$
\underline{\tilde{\Gamma}}_{H}\left(x_{1}, x_{2}, t, t-\tau\right)=\sum_{n=-\infty}^{+\infty} \underline{\tilde{\Gamma}}_{H, n}\left(x_{1}, x_{2}, \tau\right) e^{j n \omega_{0} t},
$$

with $\omega_{0}=2 \pi / T_{0}$, the tild indicating time domain representation and the fourier coefficients are given by

$$
\underline{\tilde{\Gamma}}_{H, n}\left(x_{1}, x_{2}, \tau\right)=\frac{1}{T_{0}} \int_{-T_{0} / 2}^{T_{0} / 2} \underline{\Gamma}_{H}\left(x_{1}, x_{2}, t, t-\tau\right) e^{-j n \omega_{0} t} d t,
$$

These are the cyclic time domain autocorrelation dyadics with cyclic frequencies $n f_{0}=n \omega_{0} / 2 \pi$ [12]. The cyclic correlation spectrum is then

$$
\underline{\Gamma}_{H, n}\left(x_{1}, x_{2}, \omega\right)=\int \underline{\tilde{\Gamma}}_{H, n}\left(x_{1}, x_{2}, \tau\right) e^{-j n \omega \tau} d \tau,
$$

\section{B. The measurement system}

The complexity of characterizing the EM radiation from a real complex device has led to the development of a near-field two-probe scanning system. A DUT consists of an aluminium box with an aperture of size $75 \mathrm{~mm} \times 85 \mathrm{~mm}$ is built. An Arduino Intel Galileo has been used as the source of EM emission and is placed at the centre of the aperture. The Galileo is a microcontroller designed by Intel and enrolled in the Arduino Certified program. Through this program, the Galileo is made compatible with the Arduino Software Development Environment (IDE) which allow us to program the Galileo using the Arduino software. The Galileo consists of a $400 \mathrm{MHz}$ Intel Quark SoC X1000 application processor. Measurement of a complex device means that it is impossible to provide the phase/time reference for the measurement hence the need to perform the measurement using two-probe in the time domain.

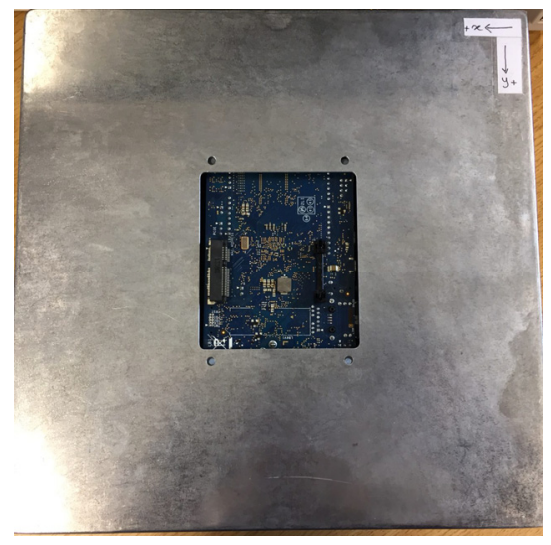

Fig. 1. DUT with the Arduino as a source of radiation.

To perform the measurement, a known program has been designed to be loaded onto the Galileo board. The program is intended to be running in a loop throughout the whole measurement process and has been tested to produce a fairly stationary EM emission from the board. In the program, random numbers are generated and will be saved in a large array in the memory. This whole program is designed so that the memory on board will be used intensively and the random elements are introduced to mimic the real data processing.

The measurement set up consists of a dual-probe 3D scanner, a PC and an $8 \mathrm{GHz}$ Keysight DSOS804a digital oscilloscope. The digital oscilloscope has 4 analogue channels that could capture a signal trace with $20 \mathrm{GSa} / \mathrm{s}$ max sample rate and 50 MPts/channel of standard memory.

The DUT is positioned underneath the scanner inside an anechoic chamber to prevent any measurement errors while the PC and digital oscilloscope is stationed on the bench outside. The whole measurement process is controlled by a LabVIEW program on the $\mathrm{PC}$ and the data at each measurement points is captured and saved to an external hard drive connected to the digital oscilloscope. Two identical Langer EMV-Technik RF R50-1 magnetic field probe of the same size $(10 \mathrm{~mm}$ in diameter), namely probe $p_{1}$ and probe $p_{2}$, are used in this measurement. The scanning plane is the size of $180 \mathrm{~mm} \times 180$ $\mathrm{mm}$ with the DUT at the centre. There are 169 measurement points with the scanning resolution of $15 \mathrm{~mm}$. 14,196 possible 
pairs of probe positions are produced from the full measurement. Two files containing time-domain data is saved at each pair of probe positions and later is used in the post processing of the data to calculate the field-field correlation.

\section{EXPLORATORY DATA ANALYSIS ON STOCHASTIC EMISSIONS NEAR-FIELD SCANNING MEASUREMENTS}

As stated in section I, the exploratory analysis of the stochastic field emissions measurements is intended to detect systematic errors within massive amounts of data. This is generated by the number of time-domain acquisitions required to complete, at least, a single voltage record of all the pairs of points defined in the scanning grid, $N_{x, y}$.

In this regard, if the number of points of the measurement grid in the $x$ and $y$ axes is $N_{x}$ and, $N_{y}$ respectively, then $N_{x, y}=N_{x} N_{y}\left(N_{x} N_{y}-1\right)$. This means, for instance, that a complete scan of a square grid of $13 \times 13$ points, produces 28,392 result files. Moreover, if we assume each result file contains an acquisition of $1 \mathrm{~ms}$ taken at a sampling frequency of $2 \mathrm{GS} / \mathrm{s}$, which is equivalent to approximately $8 \mathrm{MB} / \mathrm{file}$, the results would occupy a total file size of approximately $227 \mathrm{~GB}$. In the same vein, Fig. 2 shows an estimation of the volume of data generated by a measurement system as the one described in section II.

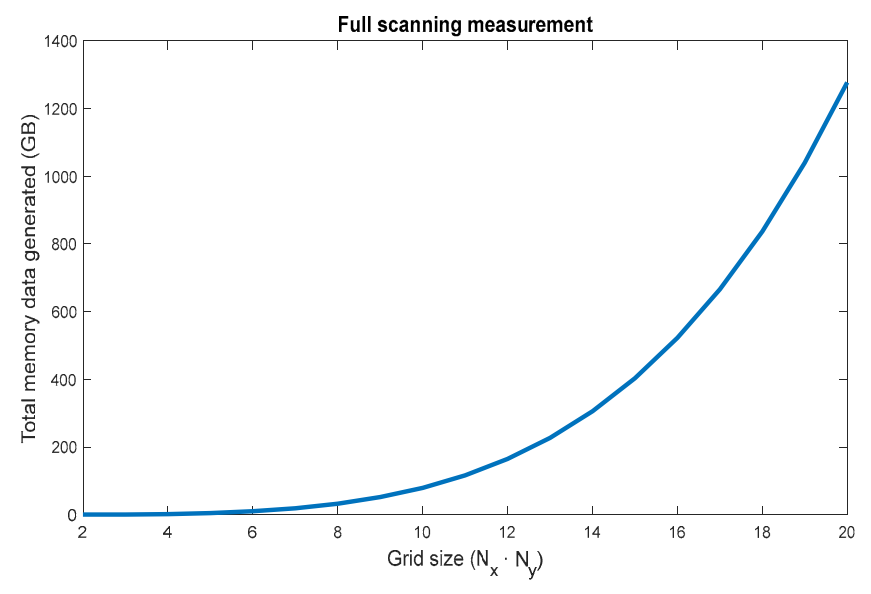

Fig. 2. The relation between memory generated and grid size.

The first step on the proposed EDA scheme is to organize the measurement data, then the sample must be selected and used for visually examining the results in a comprehensible manner and, finally, a statistical test is employed in order to make a judgement on the measurement data. Accordingly, this section presents all those three steps as follows.

\section{A. Sorting and transforming the measurements' data}

Once the full scanning measurement has been completed for a grid of $N_{x} \times N_{y}$ points, organizing the data is necessary in order to perform any further analysis. For that purpose, the approach followed is represented as a flowchart shown in Fig. 3.

First, measurement data is sorted and grouped into sequences of synchronous pairs of measurements, that is, the two acquisitions corresponding to the field probes $\left(p_{1}\right.$ and $\left.p_{2}\right)$ used on the scanning. Then, each pair of measurement is converted to the frequency domain using the Short-Time FFT and the Welch's method, with the resolution bandwidth that is in accordance with CISPR16-1-1 and with the frequency sample used on the oscilloscope for the measurements [13], [14]. Finally, the frequency spectrum of the pair of time-domain acquisitions is calculated using the Welch's method and stored. This process is iteratively repeated for all of the remaining pairs of measurement data.

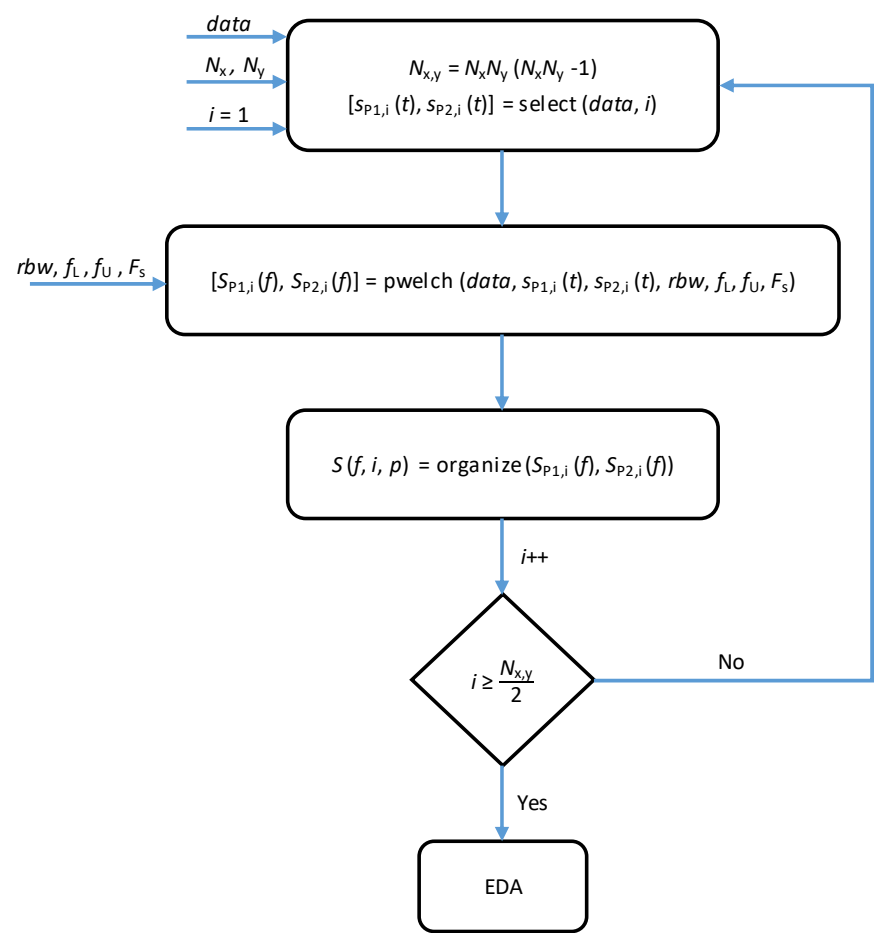

Fig. 3. Flowchart of the procedure for sorting and transforming the measurements' data.

\section{B. Selecting and exploring the sample}

Even after all measurements data has been organized and transformed into the frequency domain, there is still a lot of information to be analysed regarding the $\left(N_{x} \times N_{y}-1\right)$ frequency spectrums recorded for each point of the $N_{x} \times N_{y}$ grid. Such information is processed as following on the flowchart of Fig. 4.

Therefore, a sample of frequencies must be selected for performing the exploratory analysis. Two criterions to choose the frequencies of the sample are proposed. The sample selection criteria used in the extend of this work was to choose a number of frequencies where the maximum amplitude levels were detected according to a defined threshold and frequency deviation between each other. The alternative criteria would be to choose a number of frequencies randomly between the lower and the upper frequency range under evaluation.

Then, the distribution of each one of the selected frequencies is analysed for all the performed measurement pairs as well as for each probe used using non-parametric kernel fitting [15]. This process is repeated iteratively for all of the frequencies that are remaining, and once it is finished, the results are showed to decide if the data is valid for post-processing. 


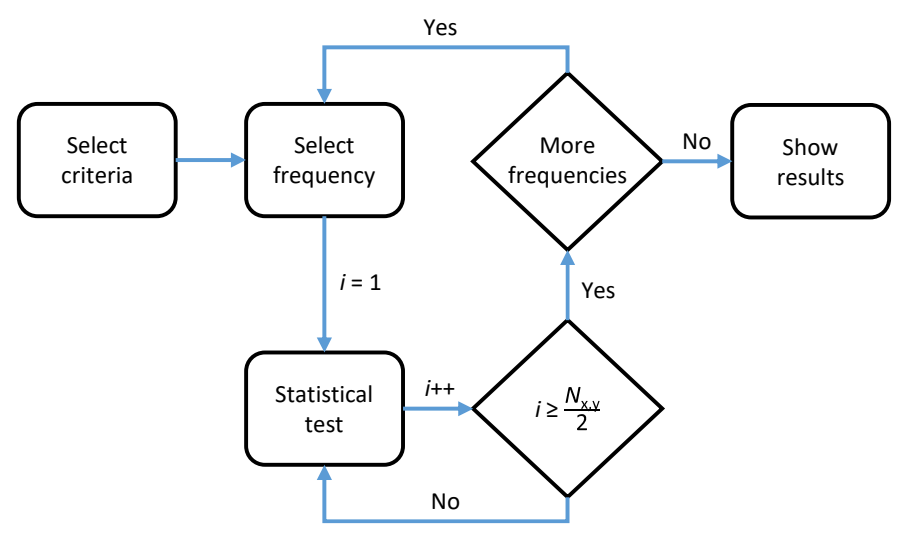

Fig. 4. Data exploration flowchart

\section{Statistical test}

The sequence of results for a given frequency of the EDA sample is examined using the Wald-Wolfowitz test, also known as the run test for randomness. The abovementioned technique is a non-parametrical statistical test used to evaluate the hypothesis that a series of numbers is random [15]. As the first step, the runs test algorithm classifies each observation above or below a specified reference value as "positive" or "negative". In that sense, the reference value could be defined as the median, the mode or the mean value, as it was done in this work.

The run test is defined as a series of consecutive positive, or negative, outcomes. There are two suppositions, the null hypothesis $H_{0}$ when the sequence was produced in a random manner, and the alternative hypothesis $H_{1}$ when the sequence was not produced in a random manner. For large sample size, the test statistic is given by,

$$
Z=\frac{R-E(R)}{S_{\mathrm{R}}}
$$

where $R$ is the observed number of runs, $E(R)$, is the expected number of runs, and $s_{\mathrm{R}}$ is the standard deviation of the number of runs. The values of $E(R)$ and $s_{\mathrm{R}}$ are computed as follows:

$$
\begin{gathered}
E(R)=\frac{2 n_{1} n_{2}}{n_{1}+n_{2}}+1, \\
S_{\mathrm{R}}=\sqrt{\frac{2 n_{1} n_{2}\left(2 n_{1} n_{2}-n_{1}-n_{2}\right)}{\left(n_{1}+n_{2}\right)^{2}\left(n_{1}+n_{2}-1\right)}},
\end{gathered}
$$

with $n_{1}$ and $n_{2}$ denoting the number of positive and negative values in the series.
Finally, the runs test rejects the null hypothesis if $|Z|>Z_{(1-\alpha / 2)}$. That is, at the $\alpha=0.05$ significance level, a test statistic with an absolute value greater than 1.96 indicates non-randomness.

\section{RESULTS}

In this section, the results of the EDA performed on a specific set of stochastic emissions near-field scanning measurements are presented as an example. In particular, a square grid of $5 \times 5$ points was used for the test. The sample was formed by 15 frequencies spaced $50 \mathrm{MHz}$ between each other, starting from $50 \mathrm{MHz}$ and ending at $750 \mathrm{MHz}$.

Due to space constrains, only the results at $400 \mathrm{MHz}$ will be shown in detail along with a final summary that includes all the frequencies of the sample. The frequency of $400 \mathrm{MHz}$ was selected because this is the processor clocks frequency of the DUT, thus detected higher emissions level were expected and detected at this frequency.

Firstly, a non-parametric distribution fitting of the amplitudes for all the measurements was performed for each point of the grid, as shown in Fig. 5. Results allow identifying two different types of distributions, the ones having a bellshaped Gaussian-like distribution on the first points and on the last points of the grid, and a multimodal distribution on the central points of the grid. Such behaviour was observed for all the examined frequencies.

Fig. 6 shows the 24 voltage measurements corresponding to the $400 \mathrm{MHz}$ frequency. Depending on the coordinates of the scanning point, the measurements are performed either with probe 1 (to the left of the vertical line) or with probe 2 (to the right of the vertical line). The green coloured ticks are used when the voltage measurement is randomly distributed around the mean voltage for the sequence of measurements corresponding to each point. Conversely, the red coloured ticks are used when the voltage measured by each field probe has a significant difference, that is, such differences are systematic. This is decided using the Wald Wolfowitz test and coincides with the analysis shown in Fig. 5. It was observed that when the majority of the measurements has been performed with a single field probe the voltage level has a similar value, but when the measurements have performed with the two field probes a systematic error is detected.

Finally, Fig. 7 shows the proportion of the frequencies that have rejected, or not, the Wald-Wolfowitz test hypothesis for all the positions of the scanning grid. The information that it gives is if the voltage variation of the measurements at each point is more likely to be due to random errors or to the intrinsic variability of the measurements (green colour) or if it is more likely to be caused by a systematic error (red colour). 

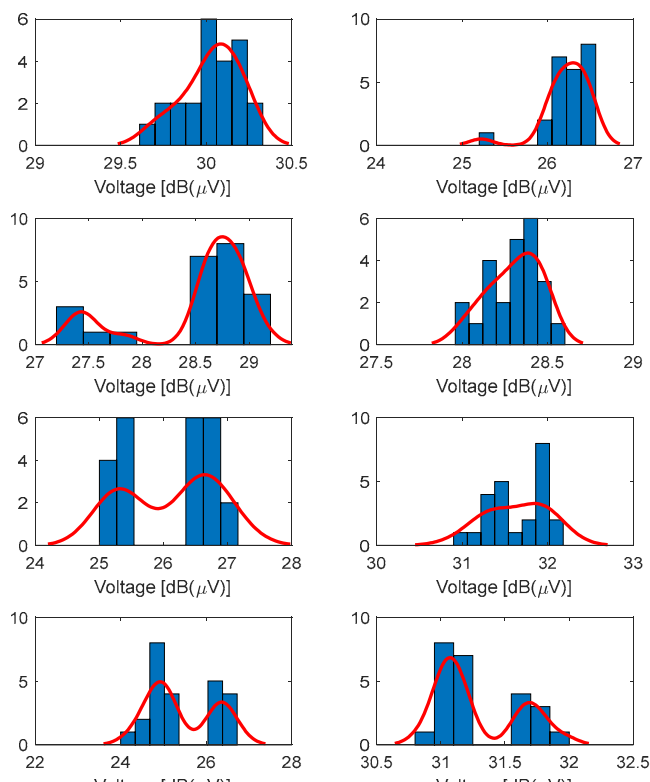

Voltage $[\mathrm{dB}(\mu \mathrm{V})]$

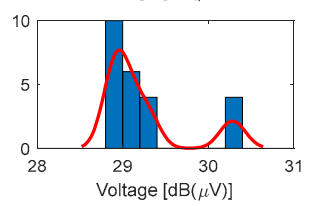

Fig. 5. Non-parametric (Kernel-fitted) distribution of the amplitudes at $400 \mathrm{MHz}$.
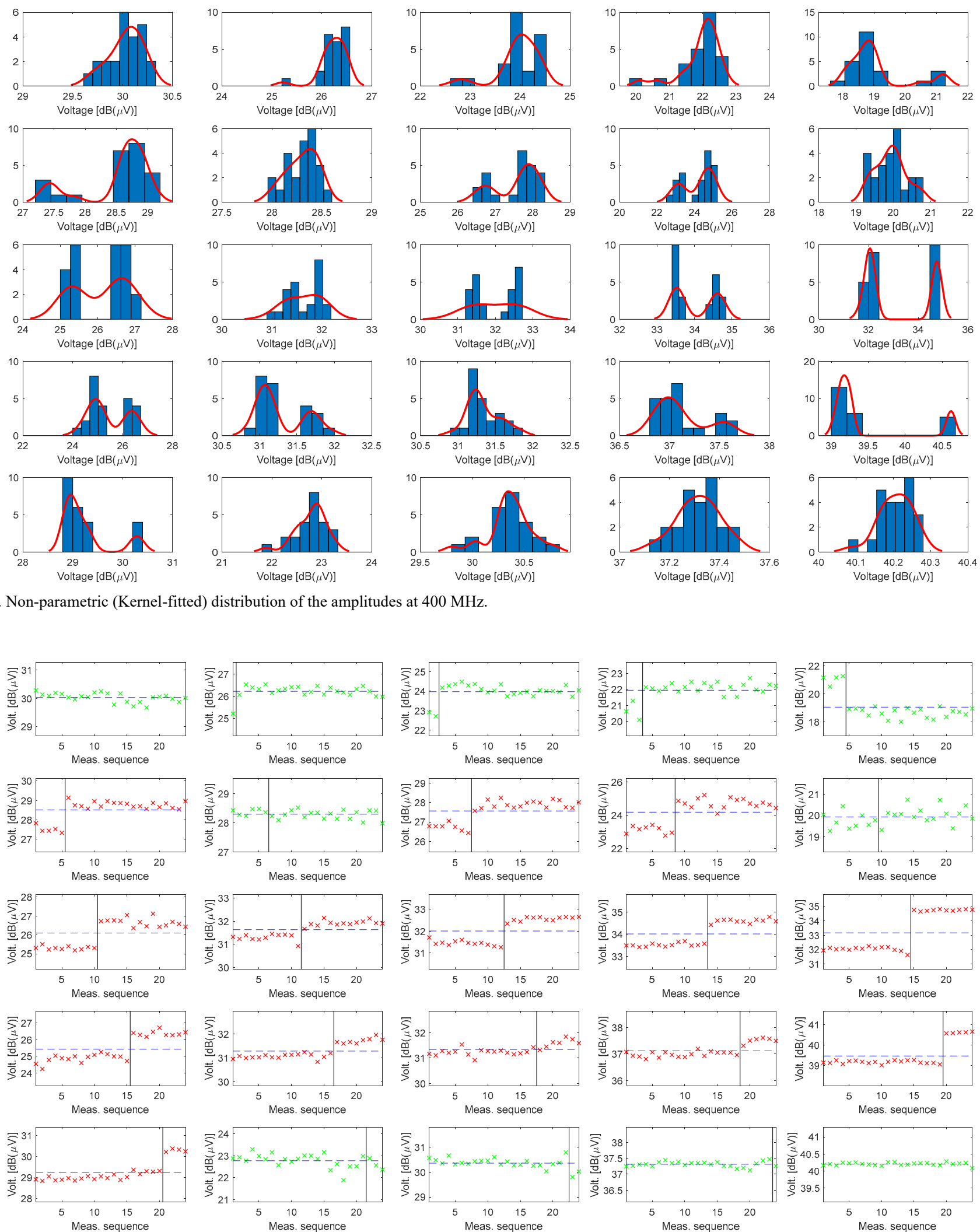

Fig. 6. Voltage level measured by probe 1 (left) and probe 2 (right) at $400 \mathrm{MHz}$. 


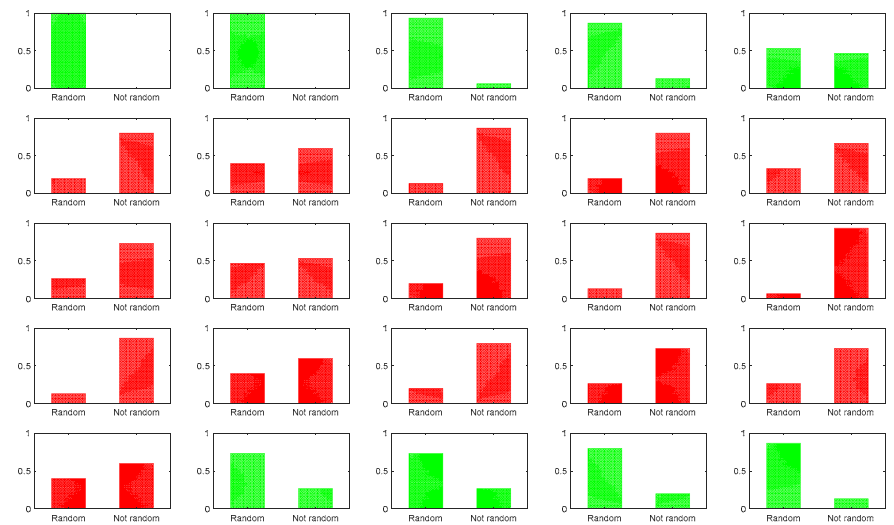

Fig. 7. The average outcome of the Wald-Wolfowitz runs test.

\section{DISCUSSION}

The multiple near-field scanning measurements are complex, time consuming and data intensive. They are not yet standardized and therefore they admit variations on the instrumentation and on the actual test setup used. This, of course, increases the likelihood of suffering from systematic errors.

For instance, the oscilloscope can be configured with different resolution bandwidth, sampling frequency, offset channels or trigger source. Moreover, the field probes and preamplifiers used in some test setups have might have a similar, but not identical, performance. In other words, each element can have a particular deviation from their typical response that must be taken into account. On the other hand, the positioners used to perform the scanning with the field probes can introduce cumulative errors in the probe position. Likewise, it is important to consider the influence on the instruments and on the DUT of such a long measurement process, and how this might affect the errors caused by, for example, temperature changes.

These different possible sources of errors and their combination can generate systematic deviations on the nearfield scanning measurements, and in the case that it passes unnoticed, an erroneous final result will be generated on the post-processing. Therefore, each contribution to the measurement uncertainty must be analysed individually in order to account for its effect on the final results.

\section{CONCLUSIONS}

Through the exploratory data analysis proposed, systematic errors have been detected on the measurements that not had been noticed previously. These systematic errors must be corrected before perform further post-processing of the data, due to the possible propagation of the systematic error to the final result. Moreover, the exact influence of these errors on the final result is unknown due to the propagation of the uncertainty on each step of the measurement process.

\section{ACKNOWLEDGMENT}

This work was supported in part by the Spanish "Ministerio de Economía, Industria y Competitividad," under project TEC2016-79214-C3-2-R (AEI/FEDER, UE) and The author(s) would like to acknowledge the contribution of the COST Action IC1407 'ACCREDIT'.

\section{REFERENCES}

[1] A. Yaghjian, "An overview of near-field antenna measurements," IEEE Trans. Antennas Propag., vol. 34, no. 1, pp. 30-45, 1986.

[2] C. A. Balanis, Advanced Engineering Mathematics. New York: John Wiley and Sons, 1989.

[3] B. Fourestie, Z. Altman, J. C. Bolomey, J. Wiart, and F. Brouaye, "Statistical modal analysis applied to near-field measurements of random emissions," IEEE Trans. Antennas Propag., vol. 50, no. 12, pp. 18031812, 2002.

[4] J. A. Russer and P. Russer, "An efficient method for computer aided analysis of noisy electromagnetic fields," in 2011 IEEE MTT-S International Microwave Symposium, 2011, pp. 1-4.

[5] C. Smartt et al., "Challenges of time domain measurement of field-field correlation for complex PCBs," in 2015 IEEE International Symposium on Electromagnetic Compatibility (EMC), 2015, pp. 953-958.

[6] G. Gradoni, S. C. Creagh, and G. Tanner, "A wigner function approach for describing the radiation of complex sources," in 2014 IEEE International Symposium on Electromagnetic Compatibility (EMC), 2014, pp. 882-887.

[7] L. R. Arnaut, C. S. Obiekezie, and D. W. P. Thomas, "Empirical Emission Eigenmodes of Printed Circuit Boards," IEEE Trans. Electromagn. Compat., vol. 56, no. 3, pp. 715-725, 2014.

[8] J. A. Russer et al., "Correlation measurement and evaluation of stochastic electromagnetic fields," in 2016 International Symposium on Electromagnetic Compatibility - EMC EUROPE, 2016, pp. 12-16.

[9] T. Li, V. Khilkevich, and D. Pommerenke, "Phase-Resolved Near-Field Scan Over Random Fields," IEEE Trans. Electromagn. Compat., vol. 58, no. 2, pp. 506-511, 2016.

[10] A. Gorbunova, A. Baev, M. Konovalyuk, and Y. Kuznetsov, "Localization of cyclostationary EMI sources based on near-field measurements," in 2015 IEEE International Symposium on Electromagnetic Compatibility (EMC), 2015, pp. 450-455.

[11] W. A. Gardner, Introduction to random process, with application to signals and systems, 2nd ed. New York: Mc Graw-Hill, 1990.

[12] J. A. Russer, P. Russer, M. Konovalyuk, A. Gorbunova, A. Baev, and Y. Kuznetsov, "Analysis of cyclostationary stochastic electromagnetic fields," in 2015 International Conference on Electromagnetics in Advanced Applications (ICEAA), 2015, pp. 1452-1455.

[13] M. A. Azpúrua, M. Pous, and F. Silva, "A measurement system for radiated transient electromagnetic interference based on general purpose instruments," in 2015 IEEE International Symposium on Electromagnetic Compatibility (EMC), 2015, pp. 1189-1194.

[14] M. A. Azpúrua, M. Pous, J. A. Oliva, B. Pinter, M. Hudlička, and F. Silva, "Waveform Approach for Assessing Conformity of CISPR 16-1-1 Measuring Receivers," IEEE Trans. Instrum. Meas., vol. PP, no. 99, pp. $1-14,2018$

[15] “NIST/SEMATECH e-Handbook of Statistical Methods,” 2012. [Online]. Available: http://www.itl.nist.gov/div898/handbook/. 